\title{
Fatores associados ao atraso vacinal em crianças menores de dois anos
}

\author{
DOI: $10.5935 / 2447-8539.201900019$
}

DEBORAH HELENA KICHESE SILVA SANTOS; LUISA SAMPAIO MACIEL; NEIDE ALVES ALMEIDA; ALVARENGA; RAPHAEL DE SOUSA DANTAS AZARIAS; PAULA FLEURY JUBÉ LEAL; VITÓRIA SANTANA DE AZEVEDO CARDOSO; RICARDO PEDROSA RIBEIRO; MAGDA REGINA SILVA MOURA

e-mail:magdasilvam@yahoo.com.br

\section{Resumo}

O monitoramento de taxas de Esquemas de Vacinação Atrasados (EVA) e das Oportunidades Perdidas de Imunização (OPI) e fatores associados é de grande importância para a saúde pública. Os objetivos desse estudo: foram calcular as taxas de EVA e OPI em crianças de 0 a 2 anos e os possíveis fatores associados, em Araguari-MG. Foram avaliados os cartões de vacinas de amostra de conveniência de 185 crianças de 0 a 2 anos em 15 Unidades de Saúde de Araguari-MG entre 01/08/2018 a 31/07/2019, por meio de um questionário sociodemográfico com informações dos responsáveis pelos menores, sobre o conhecimento de vacinas, OPI e justificativas sobre EVA. A estatística descritiva resumiu os dados. A correlação de Spearman verificou correlações entre dados sociodemográficos e EVA. OX2 verificou se existem diferenças significativas entre os dados categóricos. A taxa de EVA e OPI foram de 62,2\% e 24,9\% respectivamente. Os EVA mais frequentes foram nas doses após 12 meses de vida. As justificativas mais comuns para EVA foram o esquecimento (28,7\%) e as doenças nas crianças (27\%). Não foram encontradas correlações entre as variáveis sócio demográficas e EVA. Conclui-se que Araguari, apresenta uma taxa de EVA acima da recomendação da OMS principalmente nas doses administradas a partir de 12 meses de vida. Os fatores socioeconômicos não se associaram à EVA e a mãe foi a principal responsável pelo calendário vacinal das crianças. Os fatores associados ao atraso vacinal foram as OPI, afecções na criança no momento da aplicação da vacina e o esquecimento dos responsáveis.

Palavras-chave: Imunizações; Atraso Vacinal; Saúde da Criança.

\section{Abstract}

Monitoring rates of Delayed Vaccination Schedules (DVS) and Missed Opportunities for Vaccination (MOV) and associated factors are extremely important for public health. The aim of this study was to calculate the rates of DVS and MOV in children aged between 0 to 2 years old and their possible associated factors in the city of Araguari in MG. Here was performed the evaluation of the vaccination cards of convenience samples from 185 children aged between 0 to 2 years old in 15 Healthcare Units in Araguari between the period of 08/01/2018 and 07/31/2019. This evaluation was done via a sociodemographic questionnaire with information provided by the children's legal guardians about their knowledge regarding vaccines, MOV and justification about DVS. The descriptive statistic summarized the data. Spearman correlation verified correlations between sociodemographic data and DVS. $X^{2}$ verified the existence of significant differences between the categorical data. The DVS and MOV rates were $62.2 \%$ and $24.9 \%$, respectively. The most frequent DVS were present in the doses after the age of 12 months. The most common justifications for DVS were forgetfulness (28.7\%) and children diseases (27\%). There were no correlations between the sociodemographic variables and DVS. It is concluded that Araguari presents a DVS rate above the recommendations made by the World Health Organization (WHO), especially in the doses applied in the beginning at the age of 12 months. The socioeconomic factors were not associated to DVS and the mother was the main responsible by the vaccination calendar of their children. The factors associated to the vaccination delays were MOV, affections in the children at the moment of injecting the vaccine and forgetfulness by the legal guardians.

Keywords: Immunizations; Vaccine Delay; Child Health. 


\section{INTRODUÇÃO}

O Programa Nacional de Imunizações (PNI) é o programa que regulamenta todo o processo de vacinação no Brasil. Implantado desde 1973, transformou o panorama das imunizações até então episódicas e irregulares em um programa de referência mundial. Atualmente é um Programa da Organização Mundial da Saúde (OMS), com o apoio técnico, operacional e financeiro do Fundo das Nações Unidas para Infância (UNICEF) e contribuições do Rotary Internacional e do Programa das Nações Unidas para o Desenvolvimento (PNUD). Ele é responsável por adquirir, distribuir e normatizar o uso de agentes imunizantes (vacinas) monitora a ocorrência de enfermidades imunopreveníveis e tem como objetivo principal ofertar vacinas com qualidade à população (TEMPORÃO, 2003). Essas ações resultaram na erradicação de algumas doenças, a exemplo da varíola (1973) e a poliomielite (1989), e ainda na redução de outras como o sarampo, tétano, formas graves da tuberculose, a difteria e a coqueluche (ANDRADE, et. al, 2014; SATO, 2018; TEMPORÃO, 2003).

As vacinas são produtos biológicos que contêm substâncias como moléculas, vírus e bactérias inativados ou atenuados, que, ao serem introduzidos no organismo, estimulam o sistema imunológico na produção de anticorpos essenciais na defesa contra várias doenças infecciosas (PLOTKIN, 2008). De tal forma que o indivíduo imunizado, ao ser exposto novamente a esses microrganismos, desencadeia reações imunológicas, eliminando o agente agressor. Além disso, estimula o desenvolvimento da imunidade coletiva que consiste na aquisição de resistência de um determinado grupo ou população à introdução e disseminação de um agente infeccioso. Isso ocorre quando nessa população existe uma elevada proporção de pessoas vacinadas. Desse modo, entendem-se as ações desenvolvidas pelo como uma estratégia de grande impacto na redução da morbimortalidade por doenças infecciosas imunopreveníveis e potencialmente graves (TAVARES; RIBEIRO; OLIVEIRA, 2005; SOUSA; VIGO; PALMEIRA, 2012, PLOTKIN, 2008).

É considerada a maneira mais eficaz e segura de se prevenir doenças e reduzir morbimortalidade (TAVARES; RIBEIRO; OLIVEIRA, 2005). A importância da imunização adequada vai além de proteção individual de doenças, ela sustenta uma condição de saúde coletiva de equilíbrio com baixa prevalência de circulação desses patógenos (CONASS, 2018; RAMOS, et. al, 2018; SATO, 2018).

Estima-se que quase dois milhões de crianças ainda continuam morrendo anualmente no mundo, devido a doenças que poderiam ser evitadas através da imunização (SOUSA; VIGO; PALMEIRA, 2012). Apesar dos estudos demonstrarem queda na última década com o aumento da imunização, aleitamento materno e da melhoria das condições de vida da população, o Brasil ainda mantem altas taxas de mortalidade infantil por causa infecciosa. (FRANÇA, EB et al 2017).

O Brasil foi pioneiro na incorporação de diversas vacinas no calendário do Sistema Único de Saúde (SUS) e é um dos poucos países no mundo que ofertam de maneira universal um rol extenso e abrangente de imunobiológicos. Porém, a taxa de cobertura vacinal que sempre foi uma das principais características do $\mathrm{PNI}$, vem caindo nos últimos anos, e instaurando sinal de alerta às autoridades. 0 aumento de incidência de algumas doenças e o ressurgimento do Sarampo no Brasil e no mundo reforça essa preocupação. Embora haja uma vacina segura e custo-efetiva, em 2017 houve 110 mil mortes por sarampo no mundo, principalmente entre crianças com menos de cinco anos de idade (OPAS, 2019).

O monitoramento das taxas de cobertura de vacinação, a proporção da população com esquemas atrasados de vacinação (EVA) com relação à recomendação do PNI e as oportunidades perdidas de vacinação (OPI) são importantes indicadores que devem ser avaliados frequentemente para garantir a segurança da população (GENTILE, et. al, 2011).

Especialistas e estudiosos do Ministério da Saúde concordam que são vários os fatores que contribuem para a queda na taxa de cobertura das imunizações. Dentre esses fatores destaca-se:

1. o PNI seria vítima do seu próprio sucesso, em que doenças que desapareceram do cenário populacional não seriam mais temidas e, portanto, negligenciadas pela população e por profissionais da saúde;

2. o modelo atual de Atenção à Saúde que prioriza as afecções agudas às ações de vigilância, prevenção e promoção; e

3. diversidade de culturas e classes socioeconômicas dos familiares, fatores relacionadas a crenças, superstições, mitos e credos religiosos.

Todos esses fatores contribuem para que o esquema vacinal correto que considera o número de doses e as idades adequadas para cada vacina não seja valorizado e muitas crianças deixam de ser vacinadas (CONASS, 2018).

Outros estudos registram outras possibilidades: falta de informações das puérperas quanto à necessidade de vacinações dos recém-nascidos, desabastecimento de unidades de saúde com vacinas e em relação aos pais e responsáveis, questões culturais, descaso e outras negligências sociais (BARBIERI; COUTO; AITH, 2017, JARRET et al, 2015; LARSON et al, 2015; HORTAL; FABIO, 2019, MACDONALD, 2015). 
Outro fator a ser avaliado nesse contexto, são as Oportunidades Perdidas de Imunização (OPI). Uma OPI se estabelece quando uma pessoa, candidata à vacinação e que não possui contraindicações, visita um serviço de saúde e por qualquer motivo não recebe todas as vacinas necessárias (PAULO, 2010).

A prática de adiar a vacinação para outra consulta, a falta de disponibilidade de vacinas no local onde a necessidade de vacinar é detectada, os horários reduzidos de vacinação e as falsas contraindicações podem constituir razões importantes para as oportunidades perdidas de imunização e consequentemente redução da cobertura vacinal de uma região (ESCOBAR-DİAZ; OSORIO-MERCHAN; LAHOZ-RESTREPO, 2017; ESKOLA, 2015, GENTILE, et. al, 2011).

O Ministério da Saúde (DATASUS, 2017) registra uma cobertura vacinal do município de Araguari- MG foi de $67,37 \%$. Essa taxa que se encontra abaixo das metas preconizadas pelo PNI (varia de $80 \%$ a $100 \%$ conforme a vacina). Neste contexto, faz se necessário analisar quantitativamente a proporção de esquemas de vacinação atrasados, os fatores relacionados a esse evento e as circunstâncias que se apresentam como fatos geradores de tais atrasos.

Esse estudo se propõe a avaliar as taxas de esquemas de vacinação atrasados (EVA) e de OPI, além de avaliar os principais os fatores associados ao não cumprimento do calendário vacinal no momento oportuno em crianças de até dois anos de idade na cidade AraguariMG no período 01/08/2018 a 31/07/2019.

Espera-se que a análise das taxas de EVA e OPI seja um importante instrumento de monitoramento da avaliação da cobertura vacinal da região, fornecendo subsídios para a detecção de deficiências da cobertura vacinal, a proposição de ações preventivas e principalmente, para o norteamento de estratégias de promoção de saúde direcionadas à população, executadas no âmbito da Atenção Primária de Saúde no município.

\section{METODOLOGIA}

Trata-se de um estudo observacional, analítico, de corte transversal e de abordagem quantitativa, aprovado pelo Comitê de ética do Instituto Master de Ensino Presidente Antônio Carlos (IMEPAC) Araguari-MG sob o protocolo n 08481618.4.0000.8041 realizado no período de 01/08/2018 a 31/07/2019.

A população estudada a partir de amostra por conveniência foi composta por 185 crianças que completaram dois anos de idade no período de estudo, oriundas de 15 Unidades Básicas de Saúde da Família (UBSF) do município de Araguari-MG. Esse número representou $42,7 \%$ da população elegível o que confere uma precisão em torno de 95\% (COCHRAN, 1986).

Foi verificado nos cadastros das UBSF um total de 433 crianças que completariam dois anos no período de estudo. Foi realizada a busca ativa das crianças cadastradas nas UBSF. Foram incluídos no estudo todas as crianças identificadas e localizadas, cujos respectivos pais ou responsáveis assinaram o Termo de Consentimento Livre e Esclarecido (TCLE) durante a visita dos pesquisadores. Nenhum responsável se recusou a assinar o TCLE ou a responder a entrevista.

Foi elaborado um questionário para coleta de dados sociodemográficos do responsável e informações sobre as vacinas administradas ou não no cartão vacinal.

Quanto aos registros vacinais no cartão da criança foram avaliadas as doses registradas em relação à data de nascimento da criança e a presença de irregularidades no cumprimento do Calendário Nacional de Imunizações de 2017 de cada dose de vacinas aplicadas do nascimento até os dois anos de idade. Foi considerada vacina em atraso qualquer dose aplicada de vacina (seja única ou fracionada) fora do período preconizado para cada imunizante, ou seja, maior que 30 dias além da idade recomendada a partir da data de nascimento constante no cartão da criança.

Os dados coletados foram tabulados no software MSExcel e exportados para o software SPSS versão 25.0. Os dados descritivos foram analisados com a utilização de gráficos e tabelas e a frequência expressa por porcentagem. A amostra foi considerada paramétrica pelo teste de Shapiro-Wilk. Na análise de correlação entre as variáveis categóricas dos itens do questionário sociodemográfico "Qual o grau de instrução do responsável pelo menor?" "Qual a idade do responsável pelo menor?" "Qual a sua renda pessoal em salários mínimos?" "Qual a sua profissão?" "Qual o seu turno de trabalho?" foi utilizado o teste de correlação de Spearman.

Para avaliar a associação entre a variável vacina em atraso e motivos alegados para o atraso, a saber:

1. "Você tem dificuldade em locomover-se até a Unidade Básica de Saúde para o cumprimento do calendário vacinal?"

2. "Você considera que os profissionais de saúde sem empenham no preenchimento dos cartões de vacina?"

3. "Você alega falta de tempo ou preocupação em levar o menor a UBS?"

4. "Já ocorreu de levar seu filho a UBS e não ter disponibilidade da vacina?"

5. "Você já se esqueceu de levar o menor para se vacinar?" 
6. "Você conhece os efeitos colaterais das vacinas?"

7. "Você já deixou de vacinar a criança por causa de efeitos colaterais das vacinas?"

8. "Você considera bem informado sobre a importância de vacinação nas crianças?"

Foi utilizado o teste de Qui-Quadrado. O nível de significância adotado no estudo foi de 5\% $(p<0,05)$.

\section{RESULTADOS}

A distribuição dos participantes por Unidade de Saúde variou de 2,7 a $14,9 \%$, sendo observado um maior número de crianças na área de abrangência das Unidades de Fátima, Santa Terezinha e São Sebastião, sem significância na proporção de eventos de atrasos vacinais (Tabela 1 ).

A maioria dos respondentes eram as mães (71,9\%), 62,2\% concluíram o Ensino Médio e a faixa etária mais frequente foi entre 21 a 30 anos (42,7\%).

Tabela 1 - Demonstrativo do número e proporção de participantes por Unidade de Saúde Araguari-MG.

\begin{tabular}{lccc}
\hline \multicolumn{1}{c}{ UBSF } & $\mathrm{N}(\%)$ & Atrasos n & p valor \\
\hline Amanhece & $6,0(3,3)$ & 5,0 & \\
Bosque & $5,0(2,7)$ & 1,0 & \\
Brasília & $17,0(9,2)$ & 12,0 & \\
Chancia & $4,0(2,2)$ & 3,0 & \\
Fátima & $27,0(14,6)$ & 21,0 & \\
Goiás Parte Alta & $8,0(4,3)$ & 7,0 & $0,25^{\star}$ \\
Gutierrez & $5,0(2,7)$ & 3,0 \\
Independência & $9,0(4,9)$ & 6,0 \\
Maria Eugênia & $8,0(4,3)$ & 6,0 & \\
Miranda 1 & $9,0(4,9)$ & 6,0 \\
Miranda 2 & $9,0(4,9)$ & 6,0 \\
Novo Horizonte & $10,0(5,4)$ & 5,0 \\
Paraíso & $14,0(7,6)$ & 7,0 \\
Santa Terezinha & $27,0(14,6)$ & 12,0 \\
São Sebastião & $27,0(14,6)$ & 15,0 \\
Total & $185(100)$ & 115,0 \\
\hline
\end{tabular}

*Teste de qui quadrado $\left(x^{2}\right)$

Em relação a renda e trabalho, $48,6 \%$ dos participantes alegaram ter uma renda até 1 salário mínimo e como fonte de renda um trabalho autônomo ou liberal $(24,9 \%)$ conforme demonstrado na Tabela 2.
Quanto à declaração de conhecimento dos respondentes sobre vacinas e campanhas de vacinação, $95,1 \%$ dos respondentes afirmaram que a caderneta de vacinas da criança estava em dia no momento da entrevista e $89,2 \%$ dos pesquisados alegaram não ter dificuldades em se locomoverem até a UBSF para cumprir o calendário vacinal.

Tabela 2 - Dados sociodemográficos dos respondentes

\begin{tabular}{|c|c|c|}
\hline Variáveis & Frequência n & $\%$ \\
\hline \multicolumn{3}{|c|}{ Parentesco } \\
\hline Avós & 21,0 & 11,3 \\
\hline Irmão & 4,00 & 2,2 \\
\hline Mãe & 133,0 & 71,9 \\
\hline Pai/companheiro & 20,0 & 10,8 \\
\hline Tios & 6,00 & 3,20 \\
\hline Não declarou & 1,00 & 0,50 \\
\hline \multicolumn{3}{|c|}{ Escolaridade } \\
\hline Analfabeto & 2,0 & 1,1 \\
\hline Ensino Fundamental 1 & 5,0 & 2,7 \\
\hline Ensino Fundamental 2 & 31,0 & 16,8 \\
\hline Ensino Médio & 115,0 & 62,2 \\
\hline Ensino Superior & 27,0 & 14,6 \\
\hline Não sei/ Não quero responder & 5,0 & 2,7 \\
\hline \multicolumn{3}{|l|}{ Idade } \\
\hline Até 20 anos & 16,0 & 8,6 \\
\hline De 21 á 30 anos & 79,0 & 42,7 \\
\hline De 31 á 40 anos & 59,0 & 31,9 \\
\hline Acima de 41 anos & 29,0 & 15,6 \\
\hline Total & 183,0 & 98,9 \\
\hline Não declarou & 2,0 & 1,1 \\
\hline \multicolumn{3}{|c|}{ Renda familiar em salários mínimos (SM) } \\
\hline Até 1 & 90,0 & 48,6 \\
\hline 1 a 3 & 74,0 & 40,0 \\
\hline 3 a 6 & 19,0 & 10,3 \\
\hline 6 a 10 & 2,0 & 1,1 \\
\hline \multicolumn{3}{|l|}{ Profissão } \\
\hline Autônomo/Profissional liberal & 46,0 & 24,9 \\
\hline Estudante & 17,0 & 9,2 \\
\hline Trabalhador da Saúde & 3,0 & 1,6 \\
\hline Trabalhador de Educação & 8,0 & 4,3 \\
\hline Trabalhador do Comércio & 42,0 & 22,7 \\
\hline Trabalhador da Indústria & 20,0 & 10,8 \\
\hline Empresário & 5,0 & 2,7 \\
\hline Aposentado/Pensionista & 9,0 & 4,9 \\
\hline Desempregado & 30,0 & 16,2 \\
\hline Funcionário Público & 4,0 & 2,2 \\
\hline
\end{tabular}


A percepção dos pesquisados sobre o empenho dos profissionais das UBSF para que o calendário vacinal seja mantido em dia é positivo $(92,4 \%)$ e a maioria não alegou falta de tempo para levar a criança para a $\mathrm{s}$ UBSFs no cumprimento do calendário vacinal (85,9\%).

Quanto ao conhecimento sobre as vacinas $74,1 \%$ dos entrevistados afirmaram saber dos possíveis efeitos colaterais, contudo somente $6,5 \%$ deixaram de vacinar por causa de efeitos colaterais e $88,6 \%$ se consideram bem informados sobre a importância de se tomar as vacinas.

Foi observado que $24,9 \%$ dos respondentes afirmaram já ter comparecido a UBSF para vacinação e não ter a disponibilidade da vacina no momento da visita. Os percentuais das respostas desses itens são demonstrados no Gráfico 1.

Gráfico 1 - Percentuais das respostas dos pesquisados sobre os itens relativos á campanhas de vacinação

A caderneta de saúde da criança e/ou cartão de vacinação onde constam os registros das vacinas estão atualizadas?

Você tem dificuldade em locomover-se até a Unidade Básica de Saúde para o cumprimento do calendário vacinal?

Você considera que os profissionais de saúde sem empenharn no preenchimento dos cartões de vacina?

Você alega falta de tempo ou preocupação em levar o menor á UBS?

Já ocorreu de levar seu filho á UBS e não ter disponibilidade da vacina?

Você já se esqueceu de levar o menor para se vacinar?

Você conhece os efeitos colaterais das vacinas?

Você já deixou de vacinar a criança por causa de efeitos colaterais das vacinas?

Você considera bem informado sobre a importância de vacinação nas crianças?

॥ Sim —Não
4.9

9.7

7.6

14.1

24.9

15.1

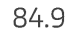

74.1

25.9

10.8

Dados perdidos
Foram registrados 556 eventos de atraso de doses vacinais em 115 (62,1\%) das 185 crianças estudadas. Dos esquemas de vacinas recomendados, a maior frequência de atrasos foi observada na segunda dose da SCR + V (Tríplice viral + varicela) em $26,6 \%$ e a que menos apresentou atraso foi a primeira dose da VHB Hepatite B (1,62\%).

Foi observado que as doses dos agentes imunizantes que mais associadas ao atraso na administração foram as que são recomendadas depois de 12 meses de vida da criança, conforme demonstrado no Gráfico 2.
As causas mais frequentemente alegadas para o atraso na aplicação da vacina foram respectivamente o esquecimento $(28,7 \%)$ e a criança estar doente na data prevista para a aplicação da dose (27\%). Todas as causas e as frequências de atraso são demonstradas no Gráfico 3.

Não foram encontradas correlações significativas entre as variáveis sócio demográfica dos respondentes e a quantidade de eventos de doses atrasadas das vacinas. Também não foram encontradas diferenças 
significativas nas proporções entre crianças com vacinas em atraso e as variáveis:

1. "Você tem dificuldade em locomover-se até a Unidade Básica de Saúde para o cumprimento do calendário vacinal?"

2. "Você considera que os profissionais de saúde se empenham no preenchimento dos cartões de vacina?"

3. "Você alega falta de tempo ou preocupação em levar o menor a UBS?"
4. "Já ocorreu de levar seu filho a UBS e não ter disponibilidade da vacina?"

5. "Você já se esqueceu de levar o menor para se vacinar?"

6. "Você conhece os efeitos colaterais das vacinas?"

7. "Você já deixou de vacinar a criança por causa de efeitos colaterais das vacinas?"

8. "Você considera bem informado sobre a importância de vacinação nas crianças?"

Gráfico 2 - Percentuais de vacinas atrasadas e em dia $(n=185)$

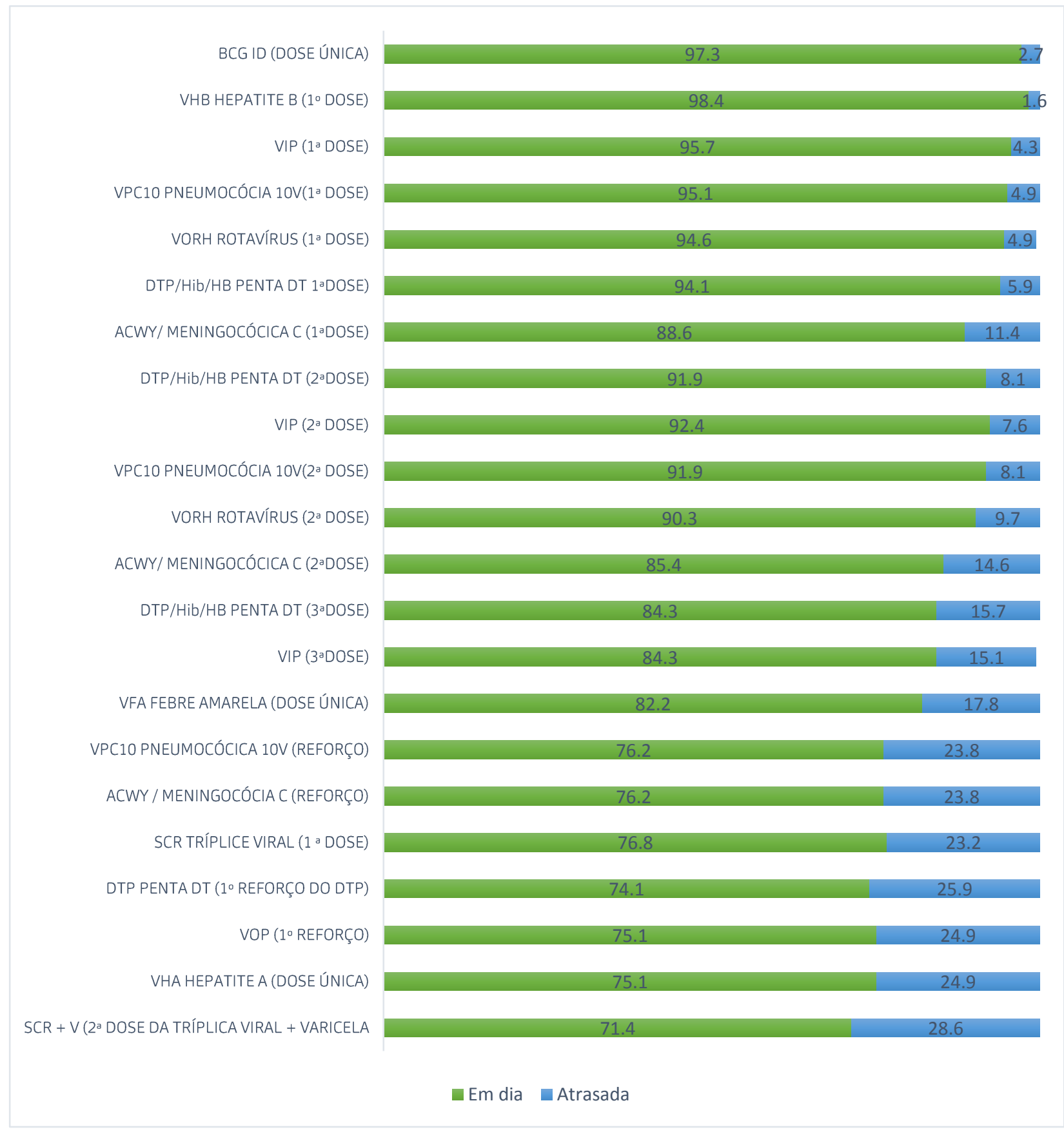


Gráfico 3 - Motivos alegados para os atrasos nas doses vacinas, em percentuais ( $n=556$ )

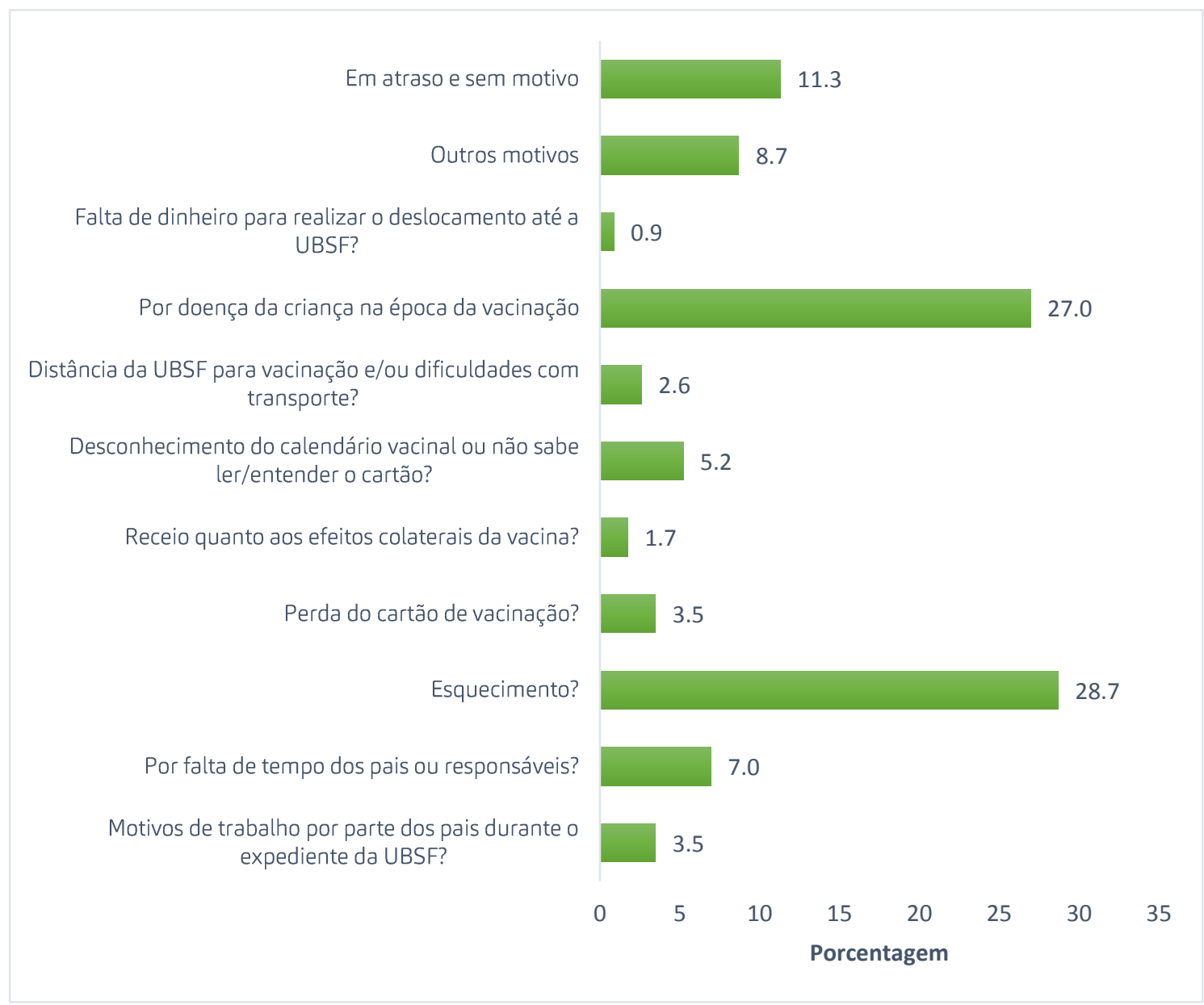

\section{DISCUSSÃO}

O presente estudo evidenciou que o município de Araguari-MG, tem uma taxa de EVA acima da recomendação da OMS, principalmente nas doses preconizadas a partir de 12 meses de vida e os fatores mais frequentemente associados ao atraso vacinal foram as OPI, as doenças nas crianças e o esquecimento dos responsáveis. Mais da metade das crianças (62,2\%) do estudo receberam pelo menos uma dose de vacina com tempo maior que 30 dias além da idade recomendada pelo calendário nacional vigente, confirmando uma taxa de EVA que extrapola os limites de até 20\% preconizados pela OMS (WORLD HEALTH ORGANIZATION, 2019).

Nesse estudo, em concordância com a literatura (TERTULIANO; STEIN, 2011, GENTILE ET AL, 2005; ANDRADE; LORENZINI; SILVA, 2014) as principais responsáveis pelo cumprimento do calendário vacinal das crianças são as mães cujas alegações para o atraso na aplicação das vacinas são atribuídas ao esquecimento e a doença dos filhos no momento da aplicação em mais da metade dos EVA.
Esse achado também foi observado no estudo de Guarda, Silva e Vilela (2018) e Barros (2015) onde as taxas de atraso foram de $20 \%$ e $40 \%$ respectivamente, mas a falta de tempo também foi outro motivo alegado pelos pais. Segundo Bujes e Silva (2012) estratégias utilizadas pelos serviços de saúde, tais como, visita domiciliar, a utilização do Dia Nacional de vacinação e meios multimídias para reforçar informações aos pais e realizar outras vacinas reduzem as taxas de EVA.

No presente estudo também foi observado que as doses de agentes imunizantes mais atrasadas geralmente, são as ministradas a partir de 12 meses de vida. Aparentemente, por serem as mais distantes do nascimento da criança, e pelo fato de boa parte delas de dose múltiplas e de reforço vacinal, induzem a um senso psicológico de tranquilidade, gerando um casual esquecimento. Outros estudos também evidenciaram alta frequência de vacinas em atraso com destaque para as vacinas com três doses ou mais com elevado percentual de atraso (PEREIRA; IVO, 2016; RAMOS et al 2010).

Segundo Paulo (2010) uma OPI se estabelece quando uma pessoa, candidata à vacinação e que não possui contraindicações, visita um serviço de saúde e por qualquer motivo não recebe todas as vacinas 
necessárias. A prática de adiar a vacinação para outra consulta, a falta de disponibilidade de vacinas no local e os horários reduzidos de vacinação aliada às falsas contraindicações podem constituir razões importantes para as oportunidades perdidas de imunização e consequentemente redução da cobertura vacinal de uma região (ESCOBAR-DİAZ; OSORIO-MERCHAN; LAHOZ-RESTREPO, 2017; ESKOLA, 2015, GENTILE, et al, 2011).

Nesse estudo as OPI foram auferidas pelo item "Já ocorreu de levar seu filho à UBS e não ter disponibilidade da vacina?" foi positivamente encontrado por aproximadamente $25 \%$ dos responsáveis.

Além disso, quase 30\% dos responsáveis alegaram doença da criança no momento da aplicação da dose. De fato algumas vacinas do esquema não são recomendadas para crianças doentes (JARRET et al, 2015; LARSON et al, 2015; HORTAL; FABIO, 2019, MACDONALD, 2015), no entanto falsas contraindicações, sendo a febre não aferida a principal delas, foi a razão de OPI mais registrada na região de São Paulo em mais de $50 \%$ da Unidades de Saúde estudadas (PAULO, 2010).

São consideradas contraindicações de vacinação: a imunodepressão, presença de doença febril moderada a grave e a reação grave de hipersensibilidade a alguma dose anterior, e que em casos de indefinição, deve ser analisado cada caso por médico competente (BARBIERI; COUTO; AITH, 2017, JARRET et al, 2015; LARSON et al, 2015; HORTAL; FABIO, 2019, MACDONALD, 2015).

Destaca-se no presente estudo a baixa proporção de responsáveis que deixaram de vacinar os filhos devido ao medo de possíveis efeitos adversos da vacina. Usualmente, o receio das vacinas se baseia em incertezas e ambiguidades que permanecem mesmo diante das mais robustas evidências científicas. Se considerarmos que os movimentos anti-vacina, embora antigos, vêm ganhando força ao longo de todo o mundo, principalmente nos países de alta renda, esse resultado é um bom prognóstico (SATO, 2018; JARRET et al, 2015; LARSON et al, 2015; HORTAL; FABIO, 2019, MACDONALD, 2015).

O estudo também evidenciou que os pais em geral se consideram bem informados e que variáveis como sexo, idade, renda e anos e escolaridade, não foram fatores de impacto nos EVA, como já registrado em outros estudos, onde a baixa escolaridade e a baixa renda se associam a EVA (ANDRADE, et. al, 2014; SATO, 2018; TEMPORÃO, 2003).

Independente dos motivos e fatores associados, atrasos nos esquemas de vacinação é um fato preocupante, pois a vacinação representa um importante aliado na diminuição da incidência e gravidade de doenças, e devido à sua efetividade e acessibilidade, é um procedimento que apresenta um menor custo que garante a promoção e proteção da saúde do indivíduo vacinado (BARBIERI; COUTO; AITH, 2017, JARRET et al, 2015; LARSON et al, 2015; HORTAL; FABIO, 2019, MACDONALD, 2015).

O Ministério da Saúde orienta algumas estratégias e ações específicas para reduzir as EVA e aumentar a taxa de cobertura vacinal:

1. Promover a capacitação e sensibilização dos profissionais para que haja um efetivo comprometimento com a vacinação das pessoas que buscam os serviços de saúde para que independente do motivo da visita seja feito uma revisão sistemática do cartão da criança; e ressaltar o valor do uso do cartão de vacinas;

2. Organizar e garantir o funcionamento efetivo do serviço de vacinação;

3. Promover ações em estabelecimento de ensino, para aprofundar o conhecimento sobre vacinação para estudantes, familiares e professores e estabelecer um planejamento para o acompanhamento sistemático do calendário de vacinação na equipe das UBSF (TERTULIANO STEIN, 2011, GENTILE et al, 2005; BRASIL, 2003).

Esse estudo apresenta limitação em alguns aspectos: não foi passivel de avaliação o horário de funcionamento das Unidades como fator limitante para os pais e não foi possível identificação das doenças que causaram o adiamento das doses vacinais. Por outro lado, o conhecimento das taxas de EVA e OPI, além de desfechos secundários como, por exemplo, identificar UBSF mais frequentes nas OPI, possibilitará às autoridades a formulação de estratégias e ações específicas para a melhoria da cobertura vacinal do município.

\section{CONCLUSÃO}

Conclui-se que o município de Araguari, apresenta uma taxa de EVA acima da recomendação da OMS principalmente nas doses administradas a partir de 12 meses de vida. Os fatores socioeconômicos e culturais não se associaram à EVA e a mãe foi a principal responsável pelo calendário vacinal das crianças.

Os fatores associados ao atraso vacinal foram as OPI, as afecções na criança no momento da aplicação da vacina e o esquecimento dos responsáveis. 


\section{REFERÊNCIAS}

ANDRADE, D. R.S; LORENZINI, E.; SILVA, E. F. Conhecimento de mães sobre o calendário de vacinação e fatores que levam ao atraso vacinal infantil. Cogitare Enfermagem, v. 19, n. 1, 2014.

BARBIERI, C. L. A.; COUTO, M. T. ; AITH, F. M. A. A (пão) vacinação infantil entre a cultura e a lei: os significados atribuídos por casais de camadas médias de São Paulo, Brasil. Cadernos de Saúde Pública, [s.l.], v. 33, n. 2, p.1-12, 2017.

BARROS, M. G.M et al. Perda de oportunidade de vacinação: aspectos relacionados à atuação da atenção primária em Recife - Pernambuco 2012. Epidemiol. Serv. Saúde, Brasília, 24(4):701-710, out-dez 2015.

BRASIL. Ministério da Saúde. Programa Nacional de Imunizações. Brasília: Ministério da Saúde; 2003.

BUJES, M.K; SILVA, J.O. Motivos do atraso vacinal em crianças e estratégias utilizadas para amenizar 0 problema - uma pesquisa bibliográfica. 26f. 2012.TCC. Universidade Federal do Rio Grande do Sul.

COCHRAN, W. Sampling Techniques. 3. ed. New York: John Wiley \& Sons, 1986.

ESCOBAR-DÍAZ, F.; OSORIO-MERCHÁN, M. B.; LAHOZRESTREPO, F. de. Motivos de no vacunación en menores de cinco años en cuatro ciudades colombianas. Revista Panamericana de Salud Pública, [s.l.], v. 41, p.1-6, 20 dez. 2017.

ESKOLA, Juhani et al. How to deal with vaccine hesitancy? Vaccine, [s.l.], v. 33, n. 34, p.4215-4217, ago. 2015.

GUARDA, K. X. da; SILVA, G. T. A. da; VILLELA, E. F. de M. Panorama da cobertura vacinal brasileira com enfoque no município de Jataí, Goiás entre 2011 e 2015. Revista de Epidemiologia e Controle de Infecção, [s.l.], v. 8, ก. 1, 12 jan. 2018.

GENTILE, A. et al, Esquemas atrasados de vacunación y oportunidades perdidas de vacunación en niños de hasta 24 meses: estudio multicéntrico, Archivos argentinos de pediatría, v. 109, p. 219-225, 2011.

HORTAL, M.; FABIO, J. L. di. Rechazo y gestión en vacunaciones: sus claroscuros. Revista Panamericana de Salud Pública, [s.l.], v. 43, p.1-5, 7 jun. 2019.

JARRETT, C. et al. Strategies for addressing vaccine hesitancy - A systematic review. Vaccine, [s.l.], v. 33, n. 34, p.4180-4190, ago. 2015.
LARSON, H. J. et al. Measuring vaccine hesitancy: The development of a survey tool. Vaccine, [s.l.], v. 33, n. 34, p.4165-4175, ago. 2015.

MACDONALD, N. E. Vaccine hesitancy: Definition, scope and determinants. Vaccine, [s.l.], v. 33, n. 34, p.4161-4164, ago. 2015.

PEREIRA, A. M.; IVO, O. P. Causas do atraso do calendário vacinal em menores de dois anos. Revista Enfermagem Contemporânea, [s.l.], v. 5, n. 2, 1 nov. 2016.

OPAS -BRASIL .Folha Informativa sobre o Sarampo . Disponível em:

https://www.paho.org/bra/index.php?option=com_con tent\&view $=$ article\&id $=5633$ :folha-informativasarampo\&ltemid=1060 . Acesso em 16/11/2019.

PLOTKIN SA, Orenstein W, Offit P. Vaccines. 5th Ed. Philadelphia: Saunders Elsevier; 2008

RAMOS, C. F. et al. Cumprimento do calendário de vacinação de crianças em uma unidade de saúde da família. Rev Pan-Amaz Saude, Ananindeua, v. 1, n. 2, p. 55-60, jun. 2010.

SATO, A. P. S. What is the importance of vaccine hesitancy in the drop of vaccination coverage in Brazil? Revista de Saúde Pública, [s.l.], v. 52, p.1-9, 22 nov. 2018.

SOUSA, C. de J.; VIGO, Z. de L.; PALMEIRA, C. S. Compreensão dos pais acerca da importância da vacinação infantil. Revista Enfermagem Contemporânea, [s.l.], v. 1, n. 1, p.45-58, 3 dez. 2012.

TAVARES, E. C.; RIBEIRO, J. G.; OLIVEIRA, L. A.. Imunização ativa e passiva no prematuro extremo. Jornal de Pediatria, [s.l.], v. 81, n. 1, p.89-94, mar. 2005.

TEMPORÃO, J. G. O Programa Nacional de Imunizações (PNI): origens e desenvolvimento. História, Ciências, Saúde-manguinhos, [s.l.], v. 10, n. 2, p.601-617, 2003.

TERTULIANO, G. C.; STEIN, A. T. Atraso vacinal e seus determinantes: um estudo em localidade atendida pela Estratégia Saúde da Família. Ciência \& Saúde Coletiva, [s.l.], v. 16, n. 2, p.523-530, fev. 2011.

WORLD HEALTH ORGANIZATION. WHO recommendations for routine immunization summary tables: - summary tables. 2019. Disponível em:

<https://www.who.int/immunization/policy/immuniza tion_tables/en/ >. Acesso em: 21 out. 2019. 